\title{
Gobernanza multidimensional del agua: la Directiva Marco del Agua europea. Dificultades de su aplicación
}

\author{
Water governance: difficulties in the application \\ of the European water framework directive
}

Resumen

Para la Unión Europea (UE) el tema de la gestión de los recursos hídricos con un enfoque de cuenca hidrológica ha sido una de las preocupaciones centrales de la problemática ambiental. Estos esfuerzos culminaron con la aprobación de la Directiva Marco del Agua (DMA) en 2000. La adhesión de nuevos países a la UE a partir del 2004 cuando pasa de la Europa de los 15 a la de los 27 en 2010, ha significado para estos últimos un esfuerzo de adaptación a todo el andamiaje legalnormativo a efecto de homologar su política de gestión y manejo hídrico. En este ensayo se enfatizan los casos de Hungría, Polonia y República Checa como países de reciente incorporación a la UE. La lección de este proceso de adhesión muestra que, si bien persisten conflictos evidentes en el uso y la aplicación de subsidios indiscriminados en el sector agrícola, déficit en el control de la calidad de aguas superficiales, así como dificultades para implementar tarifas justas tanto a usuarios agrícolas como urbanos, no obstante, se han logrado avances significativos en términos del uso y manejo sustentable del recurso.

\begin{abstract}
This paper analyses environmental issues related to application in the European Union (EU) of the Water Framework Directive, especially in countries integrated to the Union after 2004, like Poland, the Czech Republic and Hungary. These countries must comply with environmental regulations and laws applied following the principle of a common legal framework for the entire EU. There have been qualitative advances in the design and application of this Directive. However, questions and conflicts remains regarding the indiscriminate subsidies to the agricultural sector, and problems with contamination in the watersheds, as well with the implementation of fair prices and tariffs to different users of hydrological resources..
\end{abstract}

\section{Agua para la vida}

Cada tres años la onu publica el Reporte Mundial sobre el Agua, World Water Development Report (WWDR), donde se ofrece una visión estratégica y global sobre la situación y tendencias del agua potable, así como también sobre su uso por los diferentes sectores, industrial, agrícola, municipal, etc. También incluye una evaluación regional sobre disponibilidad de la misma

Keywords:

- Water Framework Directive

- European Union

- Water Sustainability

JEL: Q53 (UNESCO, 2012).

Chester (2013), citando dicho reporte señala que 780 millones de personas no tienen acceso al agua potable limpia, mientras que 2.5 mil 
millones carecen de adecuado drenaje sanitario. Estas cifras sorprendentes nos deben recordar que el tema del agua potable es un tema candente y siempre preocupante, que no sólo afecta a los países de Tercer Mundo sino que también puede afectar a países desarrollados como es el caso de la Unión Europea (UE), si bien no en la misma magnitud y dramatismo que los demás afectados. El factor del Cambio Climático (CC) le añade mayor complejidad y vulnerabilidad al problema de los recursos hídricos, su localización, escasez y manejo.

El agua constituye un recurso vital para el hombre y como tal ha ocupado y ocupa un lugar destacado dentro de la política ambiental europea, donde se ponen en juego múltiples sectores, el institucional, el socioeconómico, el financiero, el cultural y, en particular, el de la capacidad de gestión hídrica. Dentro de esto último se inscriben aspectos de reglamentación y regulación pro conservación, así como acciones de responsabilidad y reciprocidad, voluntad de pago, sistema tarifario, cánones de cobro por depuración.

El objetivo central de este ensayo es presentar los principales indicadores del desempeño hídrico-ecológico en tres países de reciente ingreso a la Unión Europea, República Checa, Hungría y Polonia, centrándonos en el análisis de la Directiva Marco del Agua (DMA) europea.

Sin duda el eje y piedra angular de la Política Ambiental Comunitaria (PAC) lo constituye la DMA aprobada el año 2000. Durante la década anterior la Unión Europea ya había elaborado importantes directrices y opiniones sobre la necesidad de abordar en el contexto más amplio de una gestión integrada de los recursos hídricos que resulte sostenible, que incluya, por ejemplo, los aspectos naturales de los sistemas de recursos hídricos, las cuencas hidrográficas, los usos del agua en todos los sectores de la economía y con cualquier fin, el marco institucional de gestión de un recurso finito, la variación espacial de los recursos y la demanda, así como la contaminación del agua (Comisión de las Comunidades Europeas, 2000).

La importancia de dicho documento reside además en otorgarle al recurso el mayor protagonismo, el cual resulta de un reconocimiento creciente de la necesidad acuciante de conseguir la seguridad global del agua, le da el imperativo de abordar la cuestión desde un punto de vista holístico y como un asunto fundamental para el desarrollo sostenible (Comisión de las Comunidades Europeas, 2000). Ahí se recogen las recomendaciones de acción formuladas en la Conferencia Internacional de Bonn (2000), proponiéndose la preparación de una iniciativa de la Unión Europea como punto clave del orden del día de la Cumbre Mundial sobre Desarrollo Sostenible. 
La Comunicación resume un enfoque exhaustivo e integrado aplicable a todos los aspectos de la gestión de los recursos hídricos y a todos los usos del agua. El mensaje básico de la orientación normativa es la preparación de estrategias basadas en los principios subyacentes de la gestión integrada de los recursos hídricos desde un enfoque de cuenca. Por otra parte, las acciones esenciales que deberán realizarse se centran en la oferta y el saneamiento del agua, su gestión transfronteriza y la coordinación e integración transectoriales. Durante todo este período la política hídrica europea no ha estado exenta del conflicto existente entre dos corrientes; la primera que privilegia el enfoque de considerar el agua como un servicio eco sistémico y de conservación de las cuencas naturales; mientras la segunda corriente, más convencional, que se apoya en las acciones del hombre, las tecnologías e infraestructuras para asegurar su abastecimiento.

De esta suerte el enfoque estratégico del acceso sostenible a los recursos hídricos y su gestión incorpora cuestiones sectoriales y transversales, afectando todos los aspectos de la sostenibilidad. En el comunicado de la UE se concluye correctamente que:

El progreso hacia un comportamiento sostenible en relación con el agua requiere el establecimiento de nuevas normas sociales, introducir la necesidad de apreciar (valorar) el agua aumentando la percepción de que es un bien precioso en todos sus usos, y buscar soluciones innovadoras y sostenibles a largo plazo, al tiempo que se reconoce que no existe una receta única que sirva para todos los problemas (Comisión de las Comunidades Europeas, 2000, p. 29).

Aunque algo tardío, este trámite de reforma integral se inicia el año 2000 con la aprobación de la DMA europea sobre el agua (Directiva 2000) y no es sino hasta el 2004 cuando entra plenamente en vigor dicha Directiva, una vez finalizado el plazo de transposición a la legislación de los estados miembros de la Unión Europea. De acuerdo con Aguilera, esta reforma se ha retrasado mucho tiempo, de hecho, la Comisión ha tardado 12 años desde que el Consejo en 1988 le solicitó la presentación de una propuesta para mejorar la calidad ecológica de las aguas de superficie de la Comunidad. Después de todo este tiempo transcurrido y aunque el texto definitivo puede ser útil, no termina, en mi opinión, de abordar de manera clara y decidida la consideración del agua como un patrimonio natural, a pesar de la declaración de intenciones (Aguilera, 2004). 


\section{Integrando la visión eco sistémica y de cuencas}

La Comisión Europea emite en 2011 una "Hoja de ruta hacia una Europa eficiente en el uso de los recursos”. Ahí se señala correctamente que el crecimiento europeo de las últimas décadas se ha basado en un uso intensivo y casi indiscriminado de los recursos naturales. De hecho el continente europeo representa el mayor importador neto de recursos (materias primas y energía) por persona (Comisión Europea, 2011, p. 6).

Esta Hoja propone evaluaciones de impacto ambiental fijando objetivos intermedios y de mediano plazo. Para ello se formulan dos niveles de indicadores núcleo provisionales:

1. Productividad de los recursos.

2. Una serie de indicadores sobre recursos básicos como son el agua, suelo, materiales, emisiones de carbono, a efecto de evaluar el consumo global de los mismos y su eficiencia.

Se considera que éste es el marco útil para enviar señales a los mercados que permitan recompensar a los productores más ecológicos, ya sea a través de medidas voluntarias u obligatorias, apoyándose también en: a) mercados líderes de la UE y; b) en la Directiva de Diseño Ecológico (Comisión Europea, 2011 , p. 5).

Considerando las tres dimensiones de la Estrategia Europa 2020 (crecimiento inteligente, sostenible e integrador), para el caso del agua se pueden resumir también en 3 los objetivos centrales de la DMA; éstos son:

1. Alcanzar un buen estado y calidad de las aguas en la UE.

2. Reducción del estrés hídrico.

3. Reducir la vulnerabilidad frente al CC.

Por su parte, el objetivo intermedio presuponía que para ese año, ya se habrán aplicado todos los planes hidrológicos de cuenca de la DMA y sólo "se recurrirá a opciones alternativas de abastecimiento de agua cuando se hayan agotado todas las posibilidades de ahorro más económicas. La extracción de agua deberá situarse por debajo de $20 \%$ de los recursos hídricos renovables que estén disponibles" (Comisión Europea, 2011, p. 16).

Del mismo modo, de manera explícita, la Comisión Europea (2012a) acepta el incumplimiento de la DMA al señalar que en Europa se despilfarra 


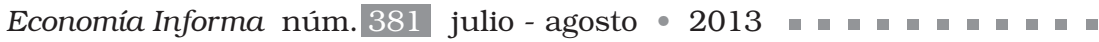

entre 20 y $40 \%$ del agua, y su consumo podría mejorar en un $40 \%$ con la sola introducción de mejoras tecnológicas, y que para el año 2015 se tendría una "situación adecuada" cubriendo los objetivos en apenas la mitad de las aguas europeas. Este objetivo se alcanzará probablemente en poco más de la mitad de las aguas de la UE (53\%). Al mismo tiempo, también se reconoce la importancia y avances de esta Directiva en la gestión del recurso:

La política de aguas de la UE ha contribuido a la protección del agua en las tres últimas décadas. Los europeos pueden beber de forma segura el agua corriente y bañarse en miles de zonas costeras, ríos y lagos de toda la UE. La contaminación, de origen urbano, industrial y agrícola es objeto de regulación, lo que ha propiciado mejoras significativas de la calidad de las aguas, particularmente reduciendo el exceso de nutrientes. Como consecuencia de ello, especies de peces emblemáticas, tales como el salmón y el esturión, han vuelto a los ríos europeos en algunas zonas (Comisión Europea, 2012a, p. 3).

Se perciben aún problemas y lagunas en la aplicación de la DMA, por ejemplo, en lo concerniente a usos excesivos, sobrexplotación de cuencas, extracción ilegal y sobre el caudal ecológico:

Para abordar el problema de la asignación excesiva, en muchas cuencas hidrográficas de la UE la gestión cuantitativa del agua debe asentarse en fundamentos más sólidos, a saber, en particular la determinación del caudal ecológico, esto es, la cantidad de agua que necesita el ecosistema acuático para seguir proporcionando los servicios indispensables. Para ello es fundamental admitir que la cantidad y la calidad del agua están estrechamente relacionadas con el concepto de "buen estado". Sin embargo, la UE no dispone de una definición de "caudal ecológico" ni existe un consenso sobre la forma en que debe calcularse, aunque ambos son requisitos necesarios para una aplicación coherente (Comisión Europea, 2012a, p. 7).

También en el informe de la Agencia Europea de Medio Ambiente (AEMA) se plantean que para el 2030 la mitad de las cuencas hidrográficas sufrirán de problemas de escasez y de estrés hídrico:

El informe de la AEMA sobre el estado de las aguas pone de relieve tendencias preocupantes que muestran el aumento y extensión mayor de la escasez de agua y del estrés hídrico, que se prevé afectarán en 2030 a aproximadamente la mitad de las cuencas hidrográficas de la UE” (Comisión Europea, 2012a, p. 11). Con respecto a las tarifas se indica que estas deben ser justas y deben cubrir al 
menos los costes de provisión así como los ecológicos de acuerdo al principio "el que contamina paga.

Por su parte, en su reporte sobre agua potable la OECD advierte correctamente que:

La clave en poner precio al agua es poder asegurar a las comunidades más pobres un acceso adecuado al sistema de suministro y los servicios de drenaje. Las experiencias sugieren que en la mitad de los países miembros de la OECD los cargos por uso de agua en hogares de bajos ingresos constituyen un tema importante o puede serlo en el futuro (...) Existen prácticas frecuentes en estos países de ampliar el acceso a los servicios de agua potable y alcantarillado focalizados a apoyar a grupos de bajos ingresos, lo cual resulta más eficiente y ambientalmente efectivo que el otorgar subsidies generalizados a través de tarifas bajas (OECD, 2008, p. 227).

Por ejemplo tarifas de agua en bloque bien diseñadas son aquellas donde los bajos consumos pagan menos y obtienen subsidios en cuotas de conexión. Comprar agua a los distribuidores de agua embotellada siempre será más costoso que pagar por la suministrada a través del servicio municipal. Otras alternativas son más onerosas en términos de los costos sociales y de oportunidad, en particular por el agua potable de calidad o el tener que recorrer largas distancias para la toma pública de agua, como ocurre en muchos países poco desarrollados.

Otro objetivo central de la DMA es que para el año 2015 todos los cuerpos de aguas superficiales en la UE deben contar con un buen estatus de calidad química y biológica. Por ejemplo los límites de concentración de plomo de 10 $\mu \mathrm{g} / 1$ establecidos por la Directiva de Agua Potable Europea de 1998 deben alcanzarse para el año 2013. Ello implica el remplazo de tuberías en los sistemas privados de suministro (OECD, 2008, p. 232).

$\mathrm{El}$ reporte antes citado parte del supuesto de que los requerimientos financieros para apoyar los sistemas de suministro de agua potable y de drenaje deben ser los siguientes:

- Para los países de altos ingresos se debe destinar entre 0.35 y $1.20 \%$ del PIB.

- Para los países de ingresos medios de 0.54 a $2.60 \%$.

- De 0.70 a $6.30 \%$ del PIB para los países de ingresos bajos. 
De manera similar, en estudios de la CEPAL desde la década de los noventa se establece que para hacer sustentables los servicios de provisión de agua potable y alcantarillado, los hogares debían destinar del orden de 3.5\% de su presupuesto total. En todo caso, se establece el principio que los sistemas de tarificación aplicados deben combinar simultáneamente ${ }^{1}$ los objetivos de eficacia con los de equidad. Su ausencia convoca a la ineficiencia en los sistemas de riego así como a onerosas obras de infraestructura hidráulica como las represas donde generalmente no se consideran los costos de las externalidades impuestas a la sociedad. De tal suerte, se puede resumir que los 3 objetivos centrales de la DMA arriba enumerados siguen siendo una asignatura pendiente y presente.

Después de un análisis y diagnostico rigurosos, el propio reporte de European Environmental, advierte en sus conclusiones que de continuar con las tendencias actuales el ciudadano europeo deberá responsabilizarse por el mal manejo del recurso hídrico:

Sin embargo un análisis detallado de los esfuerzos y logros realizados durante los 12 últimos años no nos aporta una visión optimista del futuro. Si continúan las tendencias actuales los ciudadanos europeos no se beneficiarán en el futuro inmediato con un ambiente hídrico sano; por el contrario, tendrán que cargar con la mayoría de los costos económicos y sociales derivados de la mala gestión (European Environmental Bureau, 2012a, p. 11).

Del mismo modo, podemos señalar que de las tres fases o ciclos marcados para la implementación de la Directiva, durante el primer ciclo del 2000 al 2010, no fueron aprovechadas las oportunidades que da el utilizar los instrumentos económicos. Éstos, a pesar de ser quizá los mecanismos de mercado más adecuado permitido por la legislación, no han sido aplicados de manera consecuente por la mayoría de los países miembros (European Commission, 2012, p. 2).

A la par de los indudables logros, en el informe antes citado, también se señalan los defectos y fallas del manejo hídrico integral de la UE, entre los cuales están los retrasos constantes, las excepciones y extensiones de los plazos de aplicación de la normativa del agua. Como lo mencionamos anteriormente, la agricultura aparece como el mayor contaminador, con

1 "However, economic tools don't seem to been applied well in Europe yet. As a consequence, it is still mainly households and industry that are required to pay for water use while other sectors like agriculture, mining, navigation or hydropower get away without paying their fair share" (European Commission, 2012, p. 8). 
descargas furtivas, uso extensivo de agroquímicos que afectan suelos y cuerpos de agua, con el agravante de que no cubren las tarifas necesarias.

En un estudio se señala que con la caída del Muro de Berlín muchos granjeros cayeron en bancarrota cuando sus países ingresaron a la UE y los productos subsidiados comenzaron a inundar sus mercados locales. Durante los seis primeros años, incluso muchos campesinos no calificaban para solicitar subsidios agrícolas. Ello propició la venta de granjas emergiendo una nueva élite de especuladores/inversionistas que logró adquirir vastas extensiones de tierras.

Con la Política Agrícola Común (PAC) paradójicamente el proceso de concentración de tierra y riquezas ha venido siendo apoyado a través de los subsidios con dinero público; por ejemplo en Italia en 2011 el 0.29\% de las granjas se apropiaban de $18 \%$ de los incentivos totales otorgados por la PAC y $0.0001 \%$ de aquellas (es decir, 150 granjas) se hacían de 6\% de todos los subsidios. En España durante 2009 sólo 16\% de los grandes agricultores se apropiaron de 75\% de los subsidios. En Hungría en el mismo año, 8.6\% de los granjeros se apropiaron de $72 \%$ de todos los subsidios destinados a la agricultura (Franco \& Borras, 2013, p. 114).

\section{La administración del agua en las repúblicas de Chequia, Hungría y Polonia}

En la actualidad el esquema de subsidios de la PAC se hace por la cantidad de hectáreas de cada granja. Una consecuencia inesperada de ello podría ser el acelerar el acaparamiento de tierra en la parte este y mediterránea de Europa, al marginar a los pequeños propietarios y bloquear el acceso a los subsidios por parte de los granjeros más exitosos.

Peor aún, siendo este sector el más beneficiado por los subsidios comunitarios, éstos tienden a concentrarse en pocos propietarios agricultores; en segundo término, se ha dado un proceso acelerado de acaparamiento de la tierra y agua, particularmente en los países del ex bloque socialista.

$\mathrm{Si}$ bien el acaparamiento de tierras no es un fenómeno nuevo en Hungría, éste se intensificó después de la caída del régimen comunista y su proceso de privatización a través de la entrega-venta de los llamados bonos de compensación a los propietarios agrícolas. Aquí la tierra es apreciada no sólo por su excelente calidad sino también por la abundancia de agua (Franco \& Borras, 2013, p. 116). En estos países dados los menores precios relativos del suelo con respecto al resto de Europa Occidental, la comprarenta de tierra sigue siendo un gran atractivo de inversión. 
Economía Informa núm. 381 julio - agosto • 2013 | ㅂ |

Hungría, país otrora famoso por su rica y competitiva producción cerealera, porcina, de aves de corral, de jamones, salamis y patés, hoy se debate en una fuerte caída y recesión agropecuaria. Cerca de la mitad de sus 6 millones de hectáreas de área cultivable se dedica a la producción de granos. Su participación dentro del PIB cayó de 13.7\% en 1989 a 2.5\% en 2009. También el empleo del sector primario bajó de modo sorprendente al pasar de 8.3\% en 1996 a 4.6\% en 2009 del total ocupado. No obstante, la participación de la agricultura comercial (agribusiness) dentro del PIB sigue siendo alta: entre el 12\% y 13\% en 2008 (Franco \& Borras, 2013, p. 114).

Como hemos venido señalando, el objetivo central de la política ambiental de la UE es alcanzar para el 2015 un buen estatus ecológico en los Estados Miembros (EM). Para ello se requiere cumplir con una serie de disposiciones y directivas dentro del área de calidad del agua. Al respecto una de las Directivas principales del Consejo Europeo fue la de 1991, sobre el tratamiento de aguas residuales municipales. Ésta regula tanto la colección, tratamiento como la descarga de aguas residuales urbanas y de ciertas industrias en los cuerpos de agua, evitando efectos negativos sobre el medio ambiente.

Cada dos años los países miembros deben dar un reporte sobre el "estado del arte" en el cumplimiento de las Directivas Comunitarias. De no ser así, se inicia un procedimiento denominado "proceeding on failing to fulfill the obligations", pudiendo ser multadas en caso de violaciones a las normas sobre calidad del agua (Ministry of the Environment of the Czech Republic, 2011, p. 95).

\section{Calidad del agua de los cuerpos superficiales}

De acuerdo con el Ministerio del Medio Ambiente, en la República Checa después de tres años de intensos trabajos se aprobaron en 2010 las mayores reformas del Acta del Agua a efecto de completar la implementación y ajuste con la legislación de la Comunidad Europea. Para apoyar los esfuerzos de protección y conservación, la UE destinó a este país un financiamiento por 5 mil millones de Euros para ser invertidos en el período entre 2007 y 2013 (Ministry of the Environment of the Czech Republic, 2011, p. 3).

En el país se cuenta con 5 categorías para medir el grado de contaminación de las aguas superficiales, donde las dos primeras (Iy II) son de nula o muy baja contaminación, mientras la categorías IV y V padecen alta contaminación. Según el mapa hidrológico, en la década de los noventa del siglo pasado, 
más de la mitad de las aguas superficiales se encontraban dentro de las dos últimas categorías con elevada incidencia en contaminación por pesticidas, metales pesados, coliformes fecales y cianobacterias. Para finales de 2010 el panorama se invierte con más de $50 \%$ de éstas que mejoran su calidad y pasan a las categorias I y II (Ministry of the Environment of the Czech Republic, 2011, pp. 17-18).

En los países este-europeos el porcentaje de población conectada a sistemas de redes de tratamiento de aguas residuales varía entre $52 \mathrm{y}$ $81 \%$. En la República Checa 60\% está conectado a sistemas de tratamiento terciario, mientras en Polonia alcanza a 50\% de la población y en Hungría este porcentaje sólo llega a $25 \%$ del total de tratamiento tipo terciario y el resto sólo recibe tratamiento secundario (European Environmental Agency, 2013).

Las gráficas abajo ilustran los desempeños en materia de tratamiento de aguas residuales y el porcentaje de población conectada a sistemas de drenaje de los tres países en estudio durante el período entre 1980 y 2009.

\section{Gráfica 1}

\section{Evolución en el tratamiento de aguas residuales en los países este europeos entre 1980 y 2009}

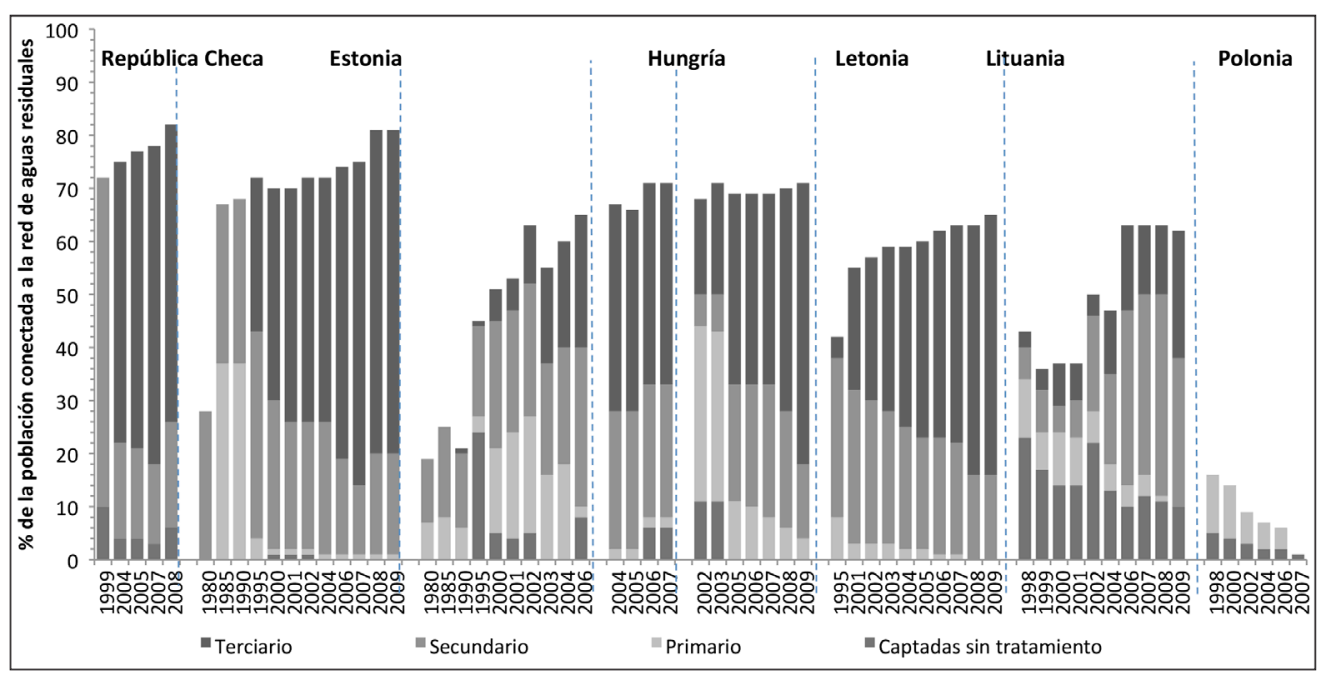

Fuente: European Environmental Agency: http://www.eea.europa.eu/data-and-maps/figures/ changes-in-wastewater-treatment-in-countries-of-europe-between-1980s-and-2005-east-2, consultado en marzo de 2013. 
Importa subrayar que las peores áreas de acuerdo a su status ecológico y las presiones por agua potable se encuentran en Europa Central y noroccidental; mientras que para zonas costeras, las peores están en las regiones del mar del Norte y el mar Báltico (European Environment Agency, 2012, pp. 8-9). Con excepción de los países escandinavos, Europa ha perdido gran parte de la biodiversidad de los ríos. Una alta responsabilidad de ello es atribuible a la construcción de presas y de plantas de generación de energía hidroeléctrica.

En Polonia la política de industrialización rápida, ignorando las afectaciones medioambientales y la sobrexplotación de los recursos naturales, colocó a este país, junto con Alemania Oriental y la Unión Soviética como uno de los más contaminados dentro del ex bloque socialista. Entre otras consecuencias, ello provocó una situación de estrés y de mala calidad del agua que padece el país.

A finales de la década de los ochenta del siglo pasado, 420 ciudades polacas no contaban con sistemas de tratamiento de descargas de aguas servidas, tanto municipales como de la industria. Las descargas se hacían directamente a los cuerpos de agua. Como consecuencia de esto el 35\% de los ríos estaban fuertemente contaminados (Council of Ministers, Republic of Poland, 2008, p. 36). Las autoridades ambientales tomaron carta en el asunto y se establecieron programas para la solución pronta de tal situación. Gracias a ello en 15 años, entre 1990 y 2005 se construyeron cerca de 3 mil plantas de tratamiento aplicando tecnologías avanzadas de depuración, dos mil de ellas en áreas rurales. Los resultados fueron que para el 2006 el 65\% de las descargas municipales se trataban ya con métodos químico-biológicos (secundario y terciario) y 27\% aplicando métodos mecánicos (Council of Ministers, Republic of Poland, 2008, p. 37). Por último se debe destacar que el programa ambiental polaco del agua estableció el año 2015 como fecha límite para cumplir con los requisitos establecidos en la Directiva Marco del Agua de la UE (Council of Ministers, Republic of Poland, 2008, p. 38).

La gráfica 2 resume el tipo de tratamiento en plantas de aguas residuales de 586 grandes ciudades/grandes descargas (con 250,2 mil p.e.) reportadas en 2011 por los países miembros. Cinco grandes ciudades reportaron no contar con ningún tratamiento de aguas residuales: 1 en Portugal, 1 en el Reino Unido y 3 en Italia. Otras siete ciudades cuentan sólo con tratamiento de tipo primario: 2 en Portugal, 1 en España, 2 en Francia y 2 en Rumanía (en todos los casos, excepto el último, el plazo para el cumplimiento de la Directiva ya había expirado). 


\section{Gráfica 2}

Tipo de tratamiento de las aguas residuales en la UE



Fuente: European Environmental Agency: http://www.eea.europa.eu/data-and-maps/ figures/number-of-eu 15-agglomerations-of-more-than-150-000-p-e-by-treatment-levelsituation-on-1st-january-1, consultado en marzo de 2013.

De la gráfica anterior se infiere un buen desempeño para 586 grandes ciudades de la Unión Europea en términos de la depuración de aguas servidas para el año 2011 , ya que $75 \%$ de su volumen total recibía tratamiento más riguroso o avanzado, mientras que $21 \%$ se sometía a un tratamiento de tipo secundario.

También en lo referente a la eficacia del tratamiento de aguas residuales municipales se cuenta con un indice diseñado por Eurostat para medirla:

- 1.00 para no tratamiento de aguas residuales,

- 0.86 para tratamiento mecánico tipo primario,

- 0.49 para tratamiento secundario adicional (biológico) y,

- 0.00 para tratamiento avanzado.

De tal suerte 100\% indica nulo tratamiento y $0.0 \%$ si todas las aguas municipales recibieran un tratamiento avanzado o terciario. Durante el período 
examinado, Hungría pasó de un índice de 80 a $0.48 \%$ mejorando 32 puntos en el porcentaje a partir de su mayor efectividad en las plantas de tratamiento de aguas residuales (Hungarian Central Statistical Office, 2012, p. 15).

Del mismo modo en la legislación Polaca se confirma el principio "el que contamina paga”, tanto para la producción de residuos como para la remoción y depuración de aguas residuales. Dicho principio se ha tratado de aplicar de manera sistemática en la política ambiental nacional (Republic of Poland, 2003).

\section{Lecciones por aprender}

Existe una correlación estrecha entre contaminación ambiental y la salud de la población y los elevados costos que ello representa. ${ }^{2}$ Enfermedades como la diarrea (gastrointestinales), alergias, neoplasias, enfermedades de las vías respiratorias, mortalidad infantil y disminución en la esperanza de vida están relacionadas también con la mala salud y el malestar de los propios ecosistemas.

El plan para la protección de los recursos hídricos señala que las aguas de la UE sufren presiones considerables relacionadas con la descarga de sustancias contaminantes, de alteraciones hidromorfológicas y de infiltración derivadas principalmente al crecimiento demográfico, al cambio de uso de suelo y a la actividad económica. Para hacer frente a estas presiones, es necesario proseguir con la aplicación de medidas de gestión de los recursos hídricos encaminadas a mejorar su eficacia y sostenibilidad, en particular:

- Medidas de retención natural de agua, para salvaguardar y fomentar el potencial de almacenamiento de agua del suelo y los ecosistemas, que ofrecen ventajas asociadas, tales como la protección de la biodiversidad, la prevención de las catástrofes naturales, la mitigación del cambio climático y la adaptación al mismo.

- Medidas de eficiencia hídrica, que a menudo constituyen un método sostenible y rentable para abordar las situaciones de estrés hídrico ya ofrecen un significativo potencial de ahorro de energía a la vez que un ahorro de agua.

- Alternativas de abastecimiento de agua, tales como su reúso, reciclaje o la desalinización.

2 Por ejemplo, la Organización Mundial de la salud estima que cada dólar invertido en el suministro, drenaje y tratamiento del agua, ahorra entre 4 y 12 dólares por gastos y costos en salud de la población (Corporate Europe Observatory, 2013). 
De conformidad con el principio de subsidiariedad, el Plan se centra en los problemas y los instrumentos estratégicos útiles para la gestión del agua a escala de la UE (Comisión Europea, 2012b, p. 3).

Sumado a lo anterior, la opción de recuperación de costos, pago por servicios eco sistémicos, el mantenimiento del caudal ecológico y medidas de retención natural de agua, son, entre otros algunos impactos ambientales (positivos) que se pueden lograr a partir de la descontaminación de cuencas y ríos, incluidos la reducción del estrés hídrico:

- El desempeño de medidas a nivel de cuenca debe tomar en cuenta los "trueques" potenciales entre, por ejemplo, retención y represamiento de aguas, versus objetivos de protección de la biodiversidad y los desplazamientos humanos.

- El adoptar medidas de eficiencia hídrica en la agricultura y en el consumo doméstico podría reducir el riesgo de estrés hídrico y el reúso de agua también contribuye a ello (European Commission, 2012, p. 55).

El tema de la descontaminación y calidad de los cuerpos de agua dentro de la UE no es un asunto menor ya que con frecuencia éstos traspasan las fronteras nacionales. La vasta red de conectividad pluvial, que incluye las cuencas marítimas requiere de una amplia cooperación territorial y macro regional.

Una lección importante digna de replicar y aprender para nuestros países es el señalamiento de la Hoja de Ruta de la UE donde fija como objetivo el año 2020 como fecha última donde ya se habrán aplicado todos los planes hidrológicos de cuenca de la Directiva Marco del Agua (DMA) y aún para el 2015 todas las cuencas fluviales de la UE deberán encontrarse en buen estado en términos de calidad, cantidad y uso (Comisión Europea, 2011, p.16). Otra meta relevante fijada por la Hoja de Ruta relacionada con la gobernanza hídrica consiste en mejorar la gestión de la demanda mediante instrumentos económicos (precios, asignación de agua) y el uso de sistemas de etiquetado y certificación que midan el impacto en todo el ciclo de vida y el contenido de agua virtual de los productos.

\section{Notas finales}

Un rápido resumen de lo analizado en este ensayo nos permite afirmar que la adhesión de estos países a la Unión Europea y la implementación de la Directiva Marco del Agua constituyó un aliciente y una palanca poderosa 


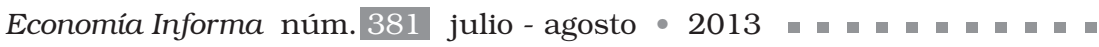

para avanzar y poner en práctica la mayoría de las normativas orientadas al medio ambiente y la protección de la naturaleza. Ello significó un paso adelante hacia la sustentabilidad y la disminución de los desequilibrios perniciosos entre la salud de los ecosistemas y el bienestar de la gente o, en todo caso, que se entienda que esto último no debe lograrse a costa del malestar y degradación de los primeros. Sin embargo, la mejoría en términos de gestión y manejo de los recursos hídricos no significa que la presión sobre los mismos haya disminuido debido, entre otras razones, a los altos niveles de consumo y demanda del ciudadano promedio europeo.

En todo caso, resulta evidente que el uso eficiente y la propia gobernanza de los recursos hídricos sólo se pueden lograr a partir de la aplicación más estricta de la legislación vigente, es decir, de la DMA, junto con la participación y corresponsabilidad ciudadanas, a partir de una nueva cultura del agua.

Lo anterior se puede inferir del informe del Buró Ambiental donde se advierte, entre otros puntos que en general la política hidrológica y sus planes no cuentan aún con las medidas necesarias para enfrentar temas como la desigual localización regional y que algunos países del sur europeo padecen ya de escasez de aguas tanto superficiales como subterráneas. Se señala también que, con pocas excepciones, para el año 2030 la mayoría de países padecerán escasez del preciado líquido, sin que existan propuestas para enfrentar el problema. De mantenerse el rumbo y actual estatus quo llevará a Europa por una senda errónea de desarrollo y costará en el futuro millones, si no es que billones de Euros a los ciudadanos. El informe concluye que si la Comisión Europea falla en la implementación de la DMA, en un futuro inmediato algunos de los ecosistemas europeos y economías que dependen del agua potable colapsarán, contribuyendo a ello las políticas de subsidios (European Environmental Bureau, 2012c, p. 2).

\section{Bibiiografía}

Aguilera, Klink F., 2004, "La Nueva Cultura del Agua", en, Aguilera, Klink F., et al. El agua en España. Propuestas de futuro, Ed. Del Oriente y del Mediterráneo, Madrid.

Chester, Penelope, 2013, "Raising Awareness With Humor On World Water Day”, sitio web consultado el 22 de marzo de 2013:

http://www.undispatch.com/raising-awareness-with-humor-on-worldwater-day.

Comisión Europea, 2011, Comunicación de la Comisión al Parlamento Europeo, al Consejo, al Comité Económico y Social Europeo y al Comité 
de las Regiones. Hoja de ruta hacia una Europa eficiente en el uso de recursos, COM (2011), 571 final. Bruselas, Documento consultado en marzo de 2013: http://eur-lex.europa.eu/LexUriServ/LexUriServ. do?uri=COM:2011:0571:FIN:ES:PDF

Comisión Europea, 2012a, Plan para salvaguardar los recursos hídricos de Europa. COM (2012) 673 final. Bruselas. Documento consultado en marzo de 2013, http://eur-lex.europa.eu/LexUriServ/LexUriServ.do?ur i=COM:2012:0673:FIN:ES:PDF.

Comisión Europea, 2012b, Plan para salvaguardar los recursos hídricos de Europa: Resumen de la evaluación de impacto, SWD (2012), 381 final. Brussels, sitio web consultado: http://eur-lex.europa.eu/LexUriServ/ LexUriServ.do?uri=SWD:2012:0381:FIN:ES:PDF.

Comisión de las Comunidades Europeas, 2000, Comunicación relativa a la política de desarrollo de la Comunidad Europea. Bruselas, COM (2000), 212, 26.4.2000.

Corporate Europe Observatory, 2013, Urban waste water treatment (CSI 024), Assessment published Jan 2013. Sitio Web consultado en marzo de 2013: http://corporateeurope.org/blog/reversing-trend-towardspublic-water

Council of Ministers, Republic of Polanda, 2008, The National Environmental Policy for 2009-2012 and its 2016 Outlook, Warsaw.

Dirección General de Desarrollo de la Comisión Europea, 2003, Hacia la Gestión Sostenible de los Recursos Hídricos: un enfoque estratégico, ECSC-EEC-EAEC, Bruselas, Luxemburgo.

Directiva 2000/60/CE, Parlamento Europeo y del Consejo, por la que se establece un marco comunitario de actuación en el ámbito de la política de aguas (conocida como DMA 2000), 23 de octubre de 2000.

European Commission, 2012, A Blueprint to Safeguard Europe's Water Resources. Impact Assessment: Part 1/2 (SWD (2012) 382 final), Brussels. Documento consultado en marzo de 2013 en: http:// ec.europa.eu/environment/water/blueprint/pdf/SWD-2012-382_ EN_impact_assessment_part1.pdf http://ec.europa.eu/environment/ water/blueprint/pdf/SWD-2012-382_EN_impact_assessment_part2.pdf

European Environmental Agency, 2012, European waters - assessment of status and pressures. Copenhagen.

European Environmental Agency, 2013, Urban Waste Water Treatment, sitio web consultado el 8 de marzo de 2013: http://www.eea.europa. eu/data-and-maps/indicators/urban-waste-water-treatment/urbanwaste-water-treatment-assessment-3. 
European Environmental Bureau, 2012a, Position on the Blueprint to Safeguard Europe's Water Resources. Bruselas. Sitio Web consultado:

http: / / www.eeb.org / ? LinkServID=40 CE 138A- 5056 - B 741 DB5084881D38E638\&showMeta=0\&aa.

European Environmental Bureau, 2012b, The EEB's Main Priorities on the Blueprint to Safeguard Europe's Water Resources. Bruselas, web consultado: http://www.eeb.org/EEB/?LinkServID=BC3165C7-5056B741-DB8C7977A71C945D\&showMeta=0.

European Environmental Bureau, 2012c, EEB recommendations for the European Council on the Blueprint to Safeguard Europe's Water Resources. Bruselas. Sitio Web consultado: http: / / www.eeb.org / ? LinkServID=F5D56E76-5056-B 741 DB6917680FB4CFE9\&showMeta=0\&aa.

Franco, Jennifer \& Saturnino M. Borras Jr. 2013, Land concentration, land grabbing and people's struggles in Europe. Transnational Institute (TNI) for European Coordination Via Campesina and Hands off the Land Network. April 2013. Documento consultado en mayo de 2013 en: http:// www.tni.org/sites/www.tni.org/files/download/land_in_europe_1.pdf

Hungarian Central Statistical Office, 2012, Environmental report, 2011. Rural Development, Agriculture and Environment Statistics Department. Budapest.

Ministry of the Environment of the Czech Republic, 2011, Report on water management in the Czech Republic in 2010. Department of Water Protection of the Czech Republic, Ministry of the Environment of the Czech Republic. Prague.

OECD, 2008, "Freshwater", in OECD Environmental Outlook to 2030, OECD Publishing. Documento consultado en marzo de 2013 en: http://dx.doi. org/10.1787/9789264040519-12-en

Republic of Poland, 2003, Water Resources Management in Poland in Environmental Context. Ministry of the Environment. Republic Of Poland, Warsaw, february 2003.

Saldívar, Américo, 2007, Las aguas de la ira, UnAM, México, D. F. Unesco, 2012, The United Nations World Water Development Report 4, Volume 1: Managing Water under Uncertainty and Risk. 